\title{
Horizontal Affordances for Patient Centred Care in Hospitals
}

\author{
Bendik Bygstad \\ University of Oslo, Halmstad University \\ bendikby@,ifi.uio.no
}

\author{
Magnus Bergquist \\ Halmstad University \\ magnus.bergquist@hh.se
}

\begin{abstract}
While it is generally accepted that patient centred care should be the guiding principle for the delivery of health services, it is not yet clear how this should be digitalised. What is clear, however, is that the current IT solutions are not satisfactory. In this research, we suggest the affordance construct as an analytical lens to understand how technological artefacts and human agency can generate action possibilities to support horizontal process innovation by asking: (i) which affordances enable digitalisation of patient centred healthcare, and (ii) how can these identified affordances be leveraged to innovate patient centred digital hospitals.

Our empirical evidence is a comparative study of two hospitals in Sweden and Norway. Our theoretical contribution is the identification of six horizontal affordances for patient centred care. The practical contribution is that horizontal affordances emerge through configurations of human actors and lightweight IT solutions, loosely coupled to heavy weight systems.
\end{abstract}

\section{Introduction}

In an editorial on person centred care, the British Medical Journal [16] wrote, "we know it improves the quality of care and promotes appropriate use of services. There is broad agreement on what it is, and guidance on how to deliver it. The challenge remains one of overcoming 'system' inertia and paternalism."

As pointed out in the editorial, hospitals are facing great challenges to make IT systems support person centred care. One important reason is that it requires a different approach than current to systems and organizational design. Today, many hospitals are dominated by systems that are organised in isolated silo structures, which are result of IT systems implemented to support specialised clinical needs. Supporting clinical needs is of course functional from the clinician's perspective, but the typical patient follows a path across organisational boundaries and require different IT systems. This constitutes both a process and a design challenge, and dealing with it requires a deep understanding of the key digitalisation issues of complex organisations.

First, we need to add a horizontal perspective to the dominant vertical (silo) specialised medical treatment regime that has dominated the current approach to digitalization in hospitals, which it shares with many other organizations [8]. For hospitals, this means that we need to focus on the needs of the patient through the care cycle, by linking clinical and logistical processes. It requires not only an improvement and integration of isolated IT systems, but a full reconceptualization of IT support for these processes.

Second, we need a deeper understanding of requirements and design. In order to support the logistical processes of such complex organisations as hospitals we cannot only ask the users in hospitals what they need. A horizontal process approach transcends the knowledge of the individual clinician or patient and needs to be supported by a rigorous method to identify causal dependencies in clinical processes.

Research on process innovation [5] and digital innovation studies [24] have contributed with important insights into a more holistic view on organisational digitalisation. In addition, Christensen [4] has linked horizontal digitalisation to health care. Kohli and Tan [10] examined the state of eHealth and concluded that integration and analytics are two key areas, that can provide the most important contribution to digitalization of hospitals. However, technology is not enough; it is the combination of technology and competent humans that can leverage health IT. There is a need to connect digital innovation more specifically to the issues of patient oriented care. In order to fill this gap, we suggest that the concept of affordance is useful. An affordance is defined as a potential for action for a goal-directed actor [22], and offers an analytical lens for a deeper understanding of how digitalisation can support patient oriented care.

With this lens, we investigate two research questions: (i) which affordances enable digitalisation 
of patient centred healthcare, and (ii) how can these affordances be leveraged to innovate patient centred digital hospitals. Our empirical evidence is a comparative case study of two illustrative, but also rather spectacular cases from hospitals in Scandinavia. From our analysis, we identify four high-level affordances.

\section{Related research: Patient centred care and digitalisation}

Patient centred care is now promoted by most national health authorities and the World Health Organization [16]. It is defined as "where health and care professional work collaboratively with people using their services, and their communities. This means it is (i) coordinated, (ii) tailored to the needs of the individual (iii) underpinned by dignity, passion and respect and enables them to lead a fulfilling life" [26]. Seen from an organisational perspective this requires a set of new competences, to identify new best practices, and to operationalise these practices in a given setting [20].

A way to operationalise them is given by Christensen [4] who built on the value configuration theory of Stabell and Fjeldstad [18], and suggested a reengineering of health care. The key point was to differentiate between the delivery of health services as a value chain, a value workshop and a value network.

Seen from a digitalisation perspective, patient oriented care can be supported both vertically and horizontally. Vertical support is typically clinical support for diagnosis, treatment or research, today supported with an increasing number of sophisticated sensors and software [19]. Horizontal support is implemented under various labels, most of them based on process thinking:

- Patient careers; the sequential steps of logistics and treatment, has been discussed since the 1960s [11].

- Patient logistics, the flow of patients through the health services [21].

- Clinical pathways, denoting the steps of treatment, seen from a medical perspective [17].

- Hospital supply chain management, a more systemic view of the flow of all types of resources [6].

- Patient oriented care, focusing on the needs of the patient, rather than the clinicians [12].

The key issue is that healthcare is generally characterised by complex clinical processes and associated silo system architecture. Much effort has been put into integrating information systems as a way to enable interoperability. This allows clinicians to access and share data across organisational borders. Although having achieved some success, these efforts have not been able to entirely overcome silo structures between organisational units. Silos do not only exist between technology architectures, but also between clinical specializations. To enable better clinical logistics in hospitals, there are several hinders that must be overcome. From an architectural perspective, an important challenge is how to maintain security and stability in clinical systems, while at the same time develop new and innovative solutions to support emerging clinical needs. From the patient-centred perspective, the challenge is how to connect different processes over organisational boundaries to prepare hospitals for patient centred care.

Adding to this challenge is the fact that both technology and clinical treatment is in the middle of an on-going revolution [19]. It is still contentious what kind of digital solutions are required to support the co-evolution of both technology structures and logistical processes to better enable clinical logistics. The approach requires an understanding of how technological artefacts and goal-oriented actors overcome the complexities generated by clinical processes, and the silo structures of IT solutions [1].

A significant part of the new IT solutions entering the e-health field has been called lightweight IT [2], denoting a combination of commercially available technology (mobile phones, apps, tablets, touchscreens) and agile implementation tactics.

\section{Analytical lens: Affordance}

Following Volkoff and Strong [22] we define an affordance from a critical realist perspective as "the potential for behaviours associated with achieving an immediate concrete outcome and arising from the relation between an object (e.g., an IT artifact) and a goal-oriented actor or actors".

In our context two aspects of affordance are important. First, it is a relational concept; it denotes neither the attributes of the technology nor the abilities of the actor, but emerges from the relation of the two [7]. For instance, a surgeon and a scalpel, together, afford the potential for a particular set of activities related to surgery.

Second, an affordance may be realized, or not. While an affordance itself is a potential for action, the details of its actualization in a specific instance are contingent on other factors, which we may group into stimulating or releasing. For instance, an 
electronic whiteboard meeting in a hospital ward may afford clinicians to take effective decisions on patient flow. However, its actualization is dependent on whether the involved nurses and doctors involve (stimulating condition) and that enough information on each patient is available to actually make the decision (releasing condition). For instance, Petrakaki et al. [13] showed that an affordance is dependent on fit between the technology and the professional's perception of it.

In our research context where we examine the horizontal processes, we differentiate between vertical and horizontal affordances. A vertical affordance enables a clinician to conduct a particular task within a defined specialisation. A horizontal affordance connects interdependent units and allows for coordinated actions.

The strength of the affordance approach is that it identifies the deeper layers of the interplay between humans and technology, compared to e.g. business process management theory. As shown in the next section we use it extensively in our methodological approach.

\section{Method}

Our research approach was a comparative, critical realist case study [23], focusing on causal explanation and middle-range theorizing.

We selected two hospitals, Halland Hospital in Sweden and Østfold Hospital in Norway. Selection criteria were, in accordance with our research questions, guided by our interest for horizontal process innovation. Both hospitals satisfied this, by being heavily involved in projects aiming to improve their horizontal processes, but with a different focus.

\subsection{Data collection}

Data was collected from a broad range of sources. We conducted in total 30 interviews with IT personnel and managers, and with clinicians and patients at the two hospitals, which is the core dataset. We also conducted several sessions with observations of meetings, practical use of technology, and of discussions between clinicians and managers. In addition, a document study was conducted at each hospital, were we gathered policy documents, project plans, evaluations, IT architecture blueprints and technical documentation.

\subsection{Data analysis}

The affordance construct provides an analytical lens to understand potentials for a particular behaviour based on the interplay of technology and human agency. In our data analysis we build on [3] in identifying and analysing affordances. Table 1 shows the steps of the process.

The study follows the critical realist method of retroduction. We started with observable events (the empirical layer) [22], which in critical realism is

Table 1. Steps in the analysis of affordances

\begin{tabular}{|c|c|c|}
\hline Step & Halland Hospital & Østfold Hospital \\
\hline 1. Description of events and issues & Case description & Case description \\
\hline 2. Identification of key entities & Clinical processes & Logistical processes \\
\hline $\begin{array}{l}\text { 3. Theoretical re-description } \\
\text { (abduction) }\end{array}$ & Value based healthcare & Value configuration \\
\hline \multicolumn{3}{|c|}{ 4. Retroduction: Identification of candidate affordances: } \\
\hline a) Identification of concrete outcomes & Live process overview of patients & Improved coordination of patients \\
\hline $\begin{array}{l}\text { b) Analysis of the interplay of human } \\
\text { and technical entities }\end{array}$ & $\begin{array}{l}\text { - Data visualisation tools } \\
\text { - Management } \\
\text { - Clinical personnel }\end{array}$ & $\begin{array}{l}\text { - Touch-screens, tablets, check-in } \\
\text { automats, mobile phones } \\
\text { - Clinical personnel }\end{array}$ \\
\hline $\begin{array}{l}\text { c) Identification of candidate } \\
\text { affordances }\end{array}$ & $\begin{array}{l}\text { - Connected clinical processes } \\
\text { - Risk prediction with machine } \\
\text { learning } \\
\text { - Improved triage }\end{array}$ & $\begin{array}{l}\text { - Self check-in for patients } \\
\text { - Touch-screen meetings for clinical } \\
\text { personnel } \\
\text { - Improved triage }\end{array}$ \\
\hline $\begin{array}{l}\text { d) Identification of stimulating and } \\
\text { releasing conditions }\end{array}$ & $\begin{array}{l}\text { - Perceived horizontal awareness } \\
\text { - Increased trust }\end{array}$ & $\begin{array}{l}\text { - Perceived usefulness } \\
\text { - Ease of use }\end{array}$ \\
\hline $\begin{array}{l}\text { 5. Analysis of high level affordances } \\
\text { and associated mechanisms }\end{array}$ & $\begin{array}{l}\text { 1. Resource optimisation } \\
\text { 2.Patient flow simulation } \\
\text { 3. Clinical learning }\end{array}$ & $\begin{array}{l}\text { 1. Visual resource planning } \\
\text { 2. Visual coordination } \\
\text { 3. Process learning }\end{array}$ \\
\hline 6. Assessment of explanatory power & $\begin{array}{l}\text { Three horizontal affordances } \\
\text { through interplay of heavy and light } \\
\text { IT }\end{array}$ & $\begin{array}{l}\text { Three horizontal affordances through } \\
\text { interplay of heavy and light IT }\end{array}$ \\
\hline
\end{tabular}


viewed as the observable subset of the actual. The actual does not need to be observable but is involved in the generation of output. As argued by e.g. Volkoff and Strong, Bygstad et al. [3,22], affordance is a way to conceptualise how structures and actors continually co-construct each other in action in a way that determines the effect of actions, i.e. the realisation of events (the mechanisms that generate the output). We studied the effects of new structures (IT-systems architectures, management discourses etc.), and the processes they generated when put into work in two hospitals. The effects were mapped by identifying events that lead to process innovation, to understand how the new processes were afforded as a property for action based on the relationship between a technical object and a user [9,22].

\section{Case Halland Hospital}

Halland Hospital serves Halland Region on Sweden's West Coast and comprises three campuses with a total of 3,800 employees and 271,000 yearly patient visits. The hospital collaborates with surrounding three university hospitals. Halland Hospital is organised in three specialist areas: medical specialities and emergency care, integrated specialist care, and clinical surgery.

\subsection{Description of events and issues}

2006: Digitalisation had gradually evolved during 1990-2000 in an ad hoc way, in response to various clinical needs. In 2006, a more systematic work starts to identify how information properties can support clinical processes. A number of processes are modelled on information resources (digital and analogue). Process support is mostly emanating from clinical needs, such as clinical learning and the request to deliver data to regional and national quality registers.

2008: The first system for electronic health records (EHR) VAS is introduced. The system is highly configurable, and existing systems are gradually integrated into the EHR.

2015: Halland Region launches a future oriented project with the aim to identify how an increasingly older population can be provided with the right level of care in the future, to control increased socioeconomically dependent health differences among inhabitants, and to cut costs. Totally some 20 projects are launched.

2015: Halland Hospital starts a collaboration with research and healthcare development teams from Brigham and Women's Hospital, Harvard Medical
School and Harvard Business School. Data sources are compiled into what will become the SHARP database.

2017: Launch of the SHARP database solution that integrates the majority of data into a data warehouse. SHARP tools are developed and initial tests on production data starts.

\subsection{Identification of key entities}

The key entities were the clinical team, external IT-consultants, the Boston research group and the ITarchitecture that combined the integrated heavyweight solution with lightweight analytic tools.

The SHARP database built on the existing VAS EHR system, to which other systems, such as ambulance, primary care, emergency ward, dentist EHR etc. gradually were connected.

SHARP implemented a clinical process thinking across organisational boundaries loosely coupled with the infrastructure. The data warehouse was created, to allow for process analyses, based on production data that followed the patient through the whole care cycle (Figure 1).

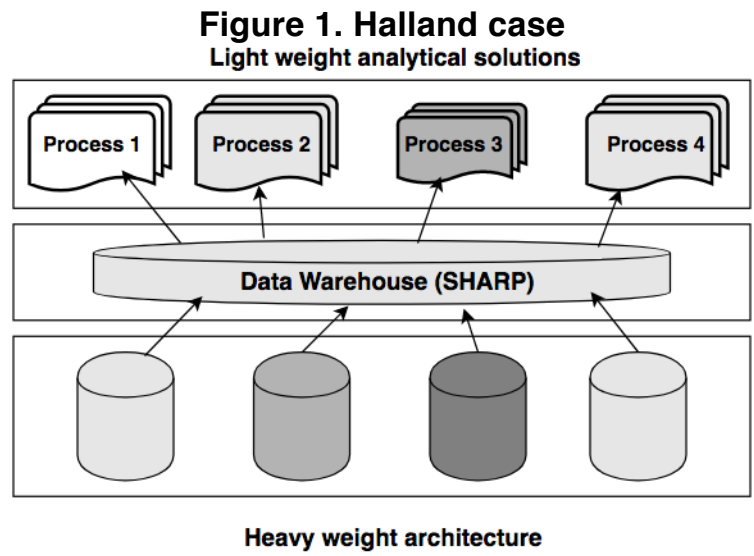

The first generation of IT architecture integrated systems into data marts divided by clinical specialisations from one or few related departments. This resulted in a process oriented mind-set within departments. The collaboration with the Boston researchers developed the approach further, and it became clear that VAS could transform into a powerful analytic tool for enterprise resource management. The most critical processes in the hospital were selected to provide maximum impact: the cancer process, the emergency ward process, the congestive heart failure process, and the patients with multiple diagnoses process. These processes offered cases with high cost ("the $20 \%$ of the population that uses $80 \%$ of the resources") and high complexity. 
The outcome of the new solution was compared to existing quality registers. The most important immediate clinical outcomes were:

- Model of the whole care cycle regardless of organisational unit or clinical specialisation.

- Personalised care trajectories based on e.g. individual, diagnose type, or other group criteria.

- Up-to-date source data directly from the production system, compared to secondary data interpreted by personnel before entered in registers.

- Deviations from optimal processes could be identified for individual patients, while existing quality registers only provided summaries of processes. Production data would identify deviations from best practice on individual level and in near real time.

- Rich data, which was flexible and adaptable to the actual situation at hand.

National quality registers were still seen as providing complementary value, since they captured information that was not supported by VAS or additional systems and allowed benchmarking with other caregivers. Together the different datasets provided a comprehensive solution for gap analysis and process modelling to streamline and improve health output. The basic approach was to identify patient journeys with most positive outcome, identify cost for each stage of the journey (lab result, x-ray, operation etc.), and improve the journeys so that the value for the patient was optimized in relation to hospital resources spent.

\subsection{Theoretical re-description: Value based healthcare}

The reconfiguration of clinical processes at Halland Hospital was inspired by the value based healthcare approach $[14,15]$. Value based healthcare defines "value for patient" measured in the outcome of activities for the benefit of the patient in relation to cost. It alters the focus from clinical orientation to patient centred care. The latter is characterised by:

- Implement an "outcome and value for patient" focus: outcomes complements measuring processes (such as practice guidelines, compliance).

- Full cycle of care: outcomes must be measured over the full cycle of care for a particular medical condition - not per intervention. This puts a focus on the entire care cycle including e.g. the time needed for recovery and the sustainability of recovery.

- Organise care around medical conditions: practice needs to be integrated to encompass skills needed to full cycle of care. More focus on prevention, screening and health maintenance. Primary care, hospital care and home care must be coordinated.

- Use electronic medical records as infrastructure: information systems should be an infrastructure to enable value improvement, i.e. support integrated care processes and outcome measurements.

Regional politicians supported the discourse on patient centred care. The project manager, a senior cardiologist who also had an engineering degree, worked with BI consultants together with the Boston group, to put the technical infrastructure in place and model the new patient centred care processes. The project manager explained:

"With this project we address all unscheduled care in Halland and can make sure that all patients enter the system on the right level, without detours or unnecessary waiting time. [...] Healthcare involves many different competences and actors. Sometimes patients meet a specialist when they really need a general practitioner. We realized that we need a comprehensive view of how patients move in the healthcare system, to certify that we use resources in the best way."

To broaden the scope and to further legitimize the project, doctors were enrolled to identify how the BI tools could be used in their practice for improved clinical learning.

\section{Case Østfold hospital}

In 1999 the Norwegian Parliament decided to build a new hospital in Østfold County. Østfold Hospital is part of the South-East Regional Health Authority, which covers around half of the Norwegian population, and has 80,000 employees. The construction at the new site started in 2010, and the hospital opened in November 2015, with both somatic and psychiatric services, and 4,800 employees.

\subsection{Description of events and issues}

The focus on processes was central from the start. In 2011 an energetic CIO was hired, with experience from production and retail, and in 2012 a Process 
Director was hired. The top management team aimed at a hospital built on process thinking and advanced IT solutions. The process challenges included for example:

- Receiving emergency patients arriving with ambulances or by taxi, registering them, conducting triage and medical diagnoses, and requiring additional services such as lab tests or radiology.

- Allocating new hospitalised patient to wards and beds, and providing the necessary information to the staff, and to patient's family.

- Setting up a clinical pathway for each patient, and allocating various resources and services in calendars

- Ensuring that each patient receives exactly the medicines that the doctor(s) had prescribed ("closed loop medication").

- Coordinating the discharge of patients with municipalities. For instance, the municipal care institutions required that information on an incoming patient should be sent before noon.

- Providing the kitchen with exact information on how many meals, dietary requirements, room numbers etc.

- Providing the cleaning department with timely information on which rooms to clean, and when.

- Creating a real-time (graphical) logistics overview for management, to ensure an optimal flow of patient and use of resources

The modelling and redesign of processes started in 2013, first in the emergency unit; later in ward. Around 25 clinicians and staff were allocated fulltime to then project, plus a number of external consultants. A work group, consisting of clinicians, IT personnel, organisation development specialists and IT architects modelled 63 work processes, each of them in considerate detail, with "swim lanes" (for roles) and the need for IT support in each step. (Most of these work processes were sub-processes of 38 different clinical pathways). A separate group worked with the details of the Imatis solution, first working with process steps; later configuring the different views of the whiteboard.

The region was at the time running an e-health mega-program, Digital Renewal, aiming at integrating the most important (silo) systems. However, the integrated package was not ready, and progress was slow. After some heated discussions in 2012 the responsibility for parts of the start-up package was transferred in 2013 from the Digital
Renewal program to the Østfold Hospital Project, because of the tight deadline in 2015 .

The Østfold project was then organised as one integrated initiative, including both physical structures, organisation and IT services. This meant that the physical architecture, the process redesign, and the supporting IT solution were all integrated in one project.

\subsection{Identification of key entities}

The key entities were the project group and clinicians in Østfold, the central IT department in Oslo, and an IT architecture that combined the regional heavyweight systems with the lightweight solution designed to support the modelled processes. See Figure 2.

The "regional package" consisted of more than 300 applications, maintained and run by the regional IT Centre. The key applications were the electronic patient record (EPR) system, lab system, radiology system and chart and medication system. Both the lab system and the chart and medication system were new, and had to be integrated into the regional package at tight deadlines. An IT architecture team was established in Østfold, particularly to deal with the complex integration issues.

A new, more lightweight IT solution was specified to support logistics and communication; a system from Imatis. This was organised as a subproject, with an external consultant as project manager, working with a group of clinicians who had modelled and redesigned many processes. The Imatis environment was strongly supported by top management, but the doctors, the IT departments, and the vendors were more sceptical.

Figure 2. Østfold case

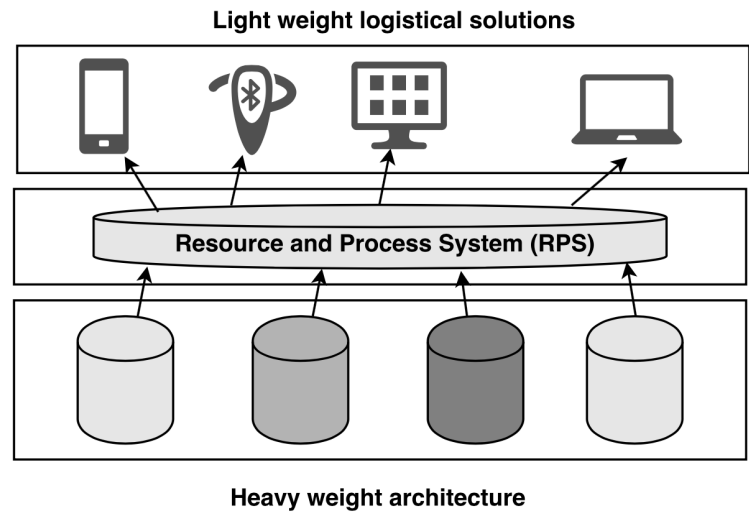




\subsection{Theoretical re-description}

The main approach in Østfold was value configuration; building on the work of Stabell and Fjeldstad [18].

The CEO of the hospital, Just Ebbesen, was a doctor and a pioneer in using IT to innovate and support clinical processes. He commented:

"I had been engaged with the relationship of process innovation and IT the past 15 years, both theoretically and practically, and I knew what I wanted to achieve: hospital processes should be well defined and supported by information. The overall thinking was inspired by the theory of value configurations (Stabell and Fjellstad, 1999), so the Moss hospital ${ }^{1}$ was designed as the value chain (dealing with the standardised high volume cases), the Østfold (Kalnes) hospital as the value workshop (dealing with complex diagnoses and treatment) we needed a comprehensive communication solution for the value network (including clinicians, staff, patients and municipalities)".

\section{Candidate affordances}

In this section, we will follow the steps of retroduction applied to both studied cases, to first identify candidate affordances for each case, and then generalise them to high level affordances. The two cases show both interesting similarities and differences. Both depict hospital process innovation driven by IT, but they also reveal important differences in how outcomes are afforded.

The study of events in each case hospital revealed two different process innovation paths (actual). At Halland Hospital clinical processes was the main focus. The Østfold Hospital case was organised around logistical processes. In Halland, EPR data was analysed to model new and more efficient processes. In the Østfold case, processes were modelled to enable IT support for new workflow. There was also different visions and conceptualisations of the projects. Halland Hospital developed IT architecture and supporting solutions based on the idea of Value based health care [15] focused on maximizing output from existing input. Østfold followed a Value configuration approach that included a division of resources between value chain, value workshop, and value network hospitals [18]. Resource coordination was thus the common denominator for Halland and Østfold hospitals.

\footnotetext{
1 The Moss Hospital was part of the Østfold Hospital, situated around 30 kilometres away.
}

Table 2 gives an overview of the identified candidate affordances.

Table 2. Candidate affordances

\begin{tabular}{|c|c|}
\hline Halland Hospital & Østfold Hospital \\
\hline 1. Real time evidence & 1. Self check-in for \\
\hline based care planning & patients \\
\hline for managers & 2. Touch-screen \\
\hline $\begin{array}{l}\text { 2. Discovery for } \\
\text { clinicians }\end{array}$ & $\begin{array}{l}\text { meeting for clinical } \\
\text { personnel }\end{array}$ \\
\hline 3. Risk prediction for & 3. Emergency \\
\hline 4. Connected clinical & resources \\
\hline processes & 4. Improved triage \\
\hline 5. Improved triage & $\begin{array}{l}\text { 5. Meal planning } \\
\text { 6. Cleaning planning }\end{array}$ \\
\hline
\end{tabular}

\subsection{Immediate outcomes}

We recognised the following outcomes. In the Halland case, structured data improved managers ability to plan more efficient patient trajectories. Clinicians could identify patient groups in a finegrained way, stratify them based on care needs and identify potential risk groups. Algorithms generated patterns of care processes, and deviations from standardised care trajectories. Second order outcomes were increased control of patient flow and better quality of treatment.

In the Østfold case the immediate outcomes were generally improved logistics, such as easier check-in for patients, less waiting and better coordination for patient flow through the various departments and services. For hospital staff outcomes were better coordination of patient flow, such as easier allocation of patient in the emergency units, and also better coordination of other resources, such as meals and room cleaning.

\subsection{Interplay of human and technical entities}

In the Halland case, managers interplayed with the data warehouse and analytic visualisation tools (BI solutions, algorithms, simulation), to make cost estimations for different care trajectories. Managers, IT-consultants and external researchers, worked with setting up the database and develop the tools, and interacted with clinicians working with selected care processes (e.g. unplanned care) to remodel care trajectories.

In the Østfold case the interplay of a two-layer IT architecture and clinicians (and other staff) provided the improved outcomes. As illustrated in Figure 2, the loosely coupled lightweight layer extracted 
information from the many heavyweight clinical systems in order to present it in the touchscreens and mobile devices. In the development phase the process modelling team worked closely with the lightweight IT vendor to provide support for each step of the processes. After implementation, the hospital established a process improvement organisation to work with continuous improvement and tuning.

\subsection{Halland candidate affordances}

The Halland Hospital solution mainly aimed at supporting clinical processes. Event analysis revealed a set of affordance candidates aimed at process support through long term strategic analysis of the organisation. Although the process "unplanned care" seems similar to the Østfold case, the solution did not aim for situated planning and short term allocation of resources. Instead, designed clinical processes pointed to long term opportunities for improvement based on careful analysis of existing processes and simulation of alternative processes based on processed real-time data. The "risk prediction for patients" affordance allowed doctors to identify possible negative outcome for specific patient groups, which could motivate alternate care plans and new research projects. The "connected clinical process" affordance generated new types of hospital wide care planning that crossed professional boundaries. The "real time evidence based care planning" affordance built legitimacy, especially for management, to plan activities using BI solutions when allocating resources.

\section{4 Østfold candidate affordances}

In the Østfold Hospital the identified affordances supported coordination, i.e. situated action and the possibility to on-demand allocation of resources in relation to shifting and unplanned tasks. For example, the "self-check-in for patients" affordance freed up personnel resources while letting the patient have more control over the hospital visit. The "touchscreen meetings for clinical personnel" affordance allowed IT to support efficient meetings by connecting daily activities to IT-based resources imported from the back-end heavy weight systems via the Resource and Process System (RPS) bus. Complex everyday tasks such as meal planning and cleaning was managed through the system as a result of decisions and task management on the interactive screen, or with a mobile phone. Some identified affordances in the Østfold case had the potential to support clinical work directly or indirectly, such as improved triage and emergency allocation of resources. However, the main bulk of actualised affordances supported resource management in situ on an everyday basis.

\subsection{Identification of high level affordances}

After carefully assessing our candidate affordances we grouped them into high level affordances that express the more general structure of the identified low level affordances. During the analysis, we identified three common aspects for both cases: resource management, patient pathway, and organisational learning. In the following section, we discuss the research questions based on the themes and their respective high level affordances. (see Table 3)

\section{Discussion}

In their commentary, Kohl and Tan [10] proposed that integration and analytics are the key approaches to support the digitalization of healthcare. We subscribe to this view, but in order to connect technology even more directly to patient centred care, we have focused on affordances.

We set out to answer two research questions: (i) which horizontal affordances enable digitalisation of patient centred healthcare, and (ii) how can these affordances be leveraged to innovate patient centred digital hospitals.

\subsection{Which horizontal affordances enable digitalisation of patient centred healthcare}

As illustrated in Table 3 we differentiate between analytical and visual affordances, where the analytical affordances are characterised by long terms strategic process development, and visual affordances enable operational coordination.

Table 3. High level affordances

\begin{tabular}{|c|c|c|}
\hline \multirow{2}{*}{ Aspect } & \multicolumn{2}{|c|}{ Patient centered care } \\
\cline { 2 - 3 } & High Level Affordances \\
\hline $\begin{array}{c}\text { Resource } \\
\text { management }\end{array}$ & $\begin{array}{c}\text { Resource } \\
\text { optimisation }\end{array}$ & $\begin{array}{c}\text { Visual resource } \\
\text { planning }\end{array}$ \\
\hline $\begin{array}{c}\text { Patient } \\
\text { pathway }\end{array}$ & $\begin{array}{c}\text { Patient flow } \\
\text { simulation }\end{array}$ & $\begin{array}{c}\text { Visual patient } \\
\text { coordination }\end{array}$ \\
\hline $\begin{array}{c}\text { Organisational } \\
\text { learning }\end{array}$ & Clinical learning & Process learning \\
\hline \multicolumn{2}{|c|}{} & \multicolumn{2}{|c|}{ Low Level Affordances } \\
\cline { 2 - 2 }
\end{tabular}


Starting with the resource management affordances, the resource optimisation affordance consists of a BI algorithm using data from many systems to enable managers to get an overview of the total cost of different clinical pathways. The visual resource planning affordance consists of visualisation of available resources from many systems that enable the clinician to take effective operational decision. Stimulating condition is a motivated decision making with a clearly identified goal. Releasing condition is that the presented information can be trusted.

For patient pathway, the patient flow simulation affordance uses simulation algorithm that enables managers to provide right level of care. The visual patient coordination affordance consists of various mobile and touchscreen technologies available throughout the hospital, allowing clinicians and patients to cooperate efficiently and create a smooth flow. Stimulating condition is the ability to act competent and informed. Releasing conditions is the availability of timely information.

In organisational learning, the clinical learning affordance consists of visualisation of dependencies between clinical processes, that allow clinicians to acquire insights in care path relations. The process learning affordance consists of process visualisations that allows clinicians and managers to assess the dependencies between processes in an operational context. Stimulating conditions is the availability of descriptions of processes. Releasing conditions is the presence of a high-level manager promoting a discourse of process thinking.

Petrakaki et al. [13] investigated the relationship and fit between affordances and professionals' cultural perceptions. We extend this knowledge by our detailed analysis of low level affordances at the hospital, which we later generalise into high level affordances.

Summing up our theoretical contribution, we offer six generic affordances and their relationship to patient centred healthcare. On a general level, we contribute to the broad discourse on process thinking in hospitals. Our resource management affordances contribute to hospital supply chain management [6]; our patient flow affordances contribute to research on patient logistics [21] and clinical pathways [17]. More specifically our affordances extend the work of Petrakaki et al. [13] by detailing the enabling relationships between technology and the user in healthcare contexts.

Our organisation learning affordances highlight that a systemic view is crucial for realizing patient centred care.

\subsection{How can these affordances be leveraged to innovate patient centred digital hospitals}

We have identified six high level affordances that we argue are important for patient centred care. All six affordances propose specific requirements for hospital IT architecture. Considering the high-level affordances, we will now discuss the consequences for IT solutions. Traditionally, IT solutions in healthcare are defined by clinicians to support patient treatment. These solutions still serve their purposes. However, from a patient centred perspective these solutions constitute technical and organisational silos, which are poorly suited to support horizontal processes.

The two studied cases reveal horizontal affordances. What are the technical solutions producing these affordances? Both solutions deal with the interplay between traditional IT silo systems and more lightweight solutions, as illustrated in Figure 1 and 2. The overall logic is that data from various IT systems are used by the lightweight systems to analyse and display processes. In the case of Halland, it was the BI solution, while in the case of Østfold, it was the visualisation solution. All six affordances were dependent on the lightweight layer.

We argue that this architecture is beneficial for providing patient centred care. A way forward for IT architectures in hospitals is a division of labour between heavyweight clinical systems and flexible lightweight solutions. To support this type of affordances there needs to be a loose coupling between the two layers.

\section{Conclusion}

In this study, we set out to investigate a horizontal view on hospital processes, focusing on patient centred care. Our research questions were, (i) which affordances enable digitalisation of patient centred healthcare, and (ii) how can these identified affordances be leveraged to innovate patient centred digital hospitals.

Our theoretical contribution is six generic affordances and their relationship to patient centred healthcare. Our practical contribution is that that we show how an IT architecture, consisting of heavyweight clinical systems, loosely coupled to lightweight IT services, is beneficial for leveraging these affordances. 


\section{References}

[1] Aanestad, M., Grisot, M., Hanseth, O., and Vassilakopoulou, P. Information Infrastructures within European Health Care. Springer, 2017.

[2] Bygstad, B. Generative innovation: a comparison of lightweight and heavyweight IT. Journal of Information Technology 32, 2 (2017), 180-193.

[3] Bygstad, B., Volkoff, O., and Munkvold, B.E. Identifying generative mechanisms through affordances: a framework for critical realist data analysis. Journal of Information Technology 31, 1 (2016), 83-96.

[4] Christensen, C.M., Grossman, J.H., and Hwang, J. The Innovator's Prescription. McGraw-Hill, 2009.

[5] Davenport, T.H. Process Innovation. Harvard Business School Press, Cambridge, MA, 1994.

[6] de Vries, H.J. and Huijsman, R. Supply chain management in health services: an overview. Supply Chain Management: An International Journal 16, 3 (2011), 159165.

[7] Faraj, S. and Azad, B. The Materiality of Technology: An Affordance Perspective. In P.M. Leonardi, B. Nardi and J. Kallinikos, eds., Materiality and Organizing. Social Interaction in a Technological World. Oxford University Press, Oxford, 2102.

[8] Gallagher, K.P., Jamey Worrell, J.L., and Mason, R.M. The negotiation and selection of horizontal mechanisms to support post-implementation ERP organizations.

Information Technology \& People 25, 1 (2012), 4-30.

[9] Hutchby, I. Technologies, Texts and Affordances. Sociology 35, 2 (2001), 441-456.

[10] Kohli, R. and Tan, S. Electronic Health Records: How Can IS Researchers Contribute to Transforming Healthcare? MIS Quarterly 40, 3 (2016), 553-573.

[11] McKinlay, J.B. The concept "patient career" as a heuristic device for making medical sociology relevant to medical students. Social Science \& Medicine 5, 5 (1967), 441-460.

[12] Meijboom, B., Schmidt-Bakx, S., and Westert, G. Supply chain management practices for improving patient oriented care. Supply Chain Management: An International Journal 16, 3 (2011), 166-175.

[13] Petrakaki, D., Klecun, E., and Cornford, T. Changes in healthcare professional work afforded by technology: The introduction of a national electronic patient record in an English hospital. Organization 23, 2 (2016), 206-226.
[14] Porter, M.E. A Strategy for Health Care Reform Toward a Value-Based System. New England Journal of Medicine 361, 2 (2009), 109-112.

[15] Porter, M.E. and Teisberg, E.O. Redefining Health Care: Creating Value-Based Competition on Results. Harvard Business Review Press, Boston, 2006.

[16] Richards, T., Coulter, A., and Wicks, P. Time to deliver patient centred care. $B M J 350$, feb10 14 (2015), h530-h530.

[17] Rotter, T., Kinsman, L., James, E.L., et al. Clinical pathways: effects on professional practice, patient outcomes, length of stay and hospital costs (Review). Cochrane Database of Systematic Reviews, (2010).

[18] Stabell, C.B. and Fjeldstad, Ø. Configuring value for competitive advantage: on chains, shops, and networks. Strategic Management Journal 19, 5 (1998), 413-437.

[19] Topol, E. The Patient Will See You Now: The Future of Medicine Is in Your Hands. Basic Books, 2016.

[20] Tucker, A.L. and Nembhard, I.M. Implementing new practices: An empirical study of organizational learning in hospital intensive care units. Management 53, 6 (2007), 894-907.

[21] van Lent, W.A., Sanders, E.M., and van Harten, W.H. Exploring improvements in patient logistics in Dutch hospitals with a survey. BMC Health Services Research 12, 1 (2012), 232-240.

[22] Volkoff, O. and Strong, D.M. Critical realism and affordances: theorizing IT-associated organizational change processes. MIS Quarterly 37, 3 (2013), 819-834.

[23] Wynn, D.J. and Williams, C.K. Principles for Conducting Critical Realist Case Study Research in Information Systems. MIS Quarterly 36, 3 (2012), 787810.

[24] Yoo, Y., Henfridsson, O., and Lyytinen, K. Research Commentary-The New Organizing Logic of Digital Innovation: An Agenda for Information Systems Research. Information Systems Research 21, 4 (2010), 724-735.

[25] Health Foundation. Person-centred care made simple. 2014. www.health.org.uk/publications/person-centred-caremade-simple. 